\title{
EDITORIALS
}

\section{Neurology and psychiatry}

'The need for collaboration between neurologist and psychiatrist is today greater than it has ever been' (Symonds, 1970). Both disciplines are devoted to behaviour and the human brain, whose most important function is mental activity. By tradition, the neurologist has concerned himself with diseases due to visible brain damage, while the psychiatrist has concentrated on mental disorder without visible underlying brain pathology. As a result of this tradition, neurology and psychiatry in the United Kingdom have separated, physically and intellectually, with the consequence that the interests of the one specialty are usually not available or used by the other. The mental disorders produced by diseases of the brain abound in neurological and neurosurgical wards, yet few psychiatrists are in close enough contact to make use of this material to further their own subject. Similarly, the effects of the drugs employed routinely in psychiatry on brain function are of great theoretical importance to neurology, yet few neurologists are in a position to make use of this opportunity.

There are fields of common interest to both disciplines which have always been, and continue to be, productive. Epilepsy is an illness which breaks the traditional division between neurologist and psychiatrist -an illness with frequent mental disorder and visibly normal brain, but due to a functional abnormality of cerebral physiology. Epilepsy taught neurologists much about the physiology of the human brain and psychiatrists something about the relation of mental disorder to nervous function. The effect of surgery of temporal lobe epilepsy on behaviour, as well as seizures, has proved a fruitful field of investigation for the psychiatrist, while the effect of anticonvulsant drugs on mood, behaviour, and drive needs to be established. Since the practical management of the epileptic patient often involves both psychiatrist and neurologist, the opportunities for conjoint study of common problems exist, the more so if the proposals of the Reid report are to be implemented widely. Dementia is another problem shared by both disciplines. Hitherto, the study of dementia has not yielded great practical reward. However, both psychiatric and neurological study in recent years give promise for further advance. The definition in clinical and pathological terms of the common dementing illness has paved the way for fundamental study. The recognition of the entity of lowpressure communicating hydrocephalus has added another treatable condition to the list of causes of dementia, while the discovery that Creutzfeldt-Jakob disease is transmissible to primates has opened up a new field of understanding of so-called degenerative conditions and may lead to effective treatment in the future.

Perhaps the most dramatic advance in neurology in the last decade has been the use of levodopa to treat Parkinson's disease, and this discovery originally stemmed from psychiatry. The observation that certain drugs, introduced into psychiatric practice in the 1950s to treat schizophrenia, caused a Parkinsonism syndrome as a side-effect led to the pharmacological discoveries that culminated in the dramatic success of levodopa treatment. There is a curious similarity between the evolution of the discovery that dopamine deficiency underlies Parkinson's disease and the presently fashionable 'catacholamine' hypotheses of affective illness. Neurologists are convinced that loss of the dopaminergic nigrostriatal tract is responsible for most of the disability of Parkinson's disease, while psychiatrists are working on the notion that an excess or a deficiency of monoamine activity in the brain underlies mania and depression. Amines again enter in the field of schizophrenia, where it is suggested that the efficacy of drugs used to control schizophrenia depends on their ability to alter the disposition of cerebral dopamine-the monoamine which to neurologists is synonymous with Parkinson's disease. While neurologists extend their investigations of brain amine metabolism to try to explain abnormal movement disorders, such as chorea or torsion dystonia, psychiatrists explore the relation of the same amines to the major psychoses. The outsider must conclude that either one team or the other is 
barking up the wrong tree, or that there is a fundamental common link between the two groups of disease. If a neurologist might be allowed to speculate, could it be that the basal ganglia, perhaps aptly termed the last cerebral virgins, are just as concerned with emotion, thought, and intellect, as with motor control?

Aside from such speculation, the activities of each of the two disciplines in this field of common endeavour are of considerable interest to the other. Neurologists are now armed with relatively specific direct stimulants of brain noradrenergic and dopaminergic receptors. They will use these tools to try and refine the treatment of Parkinson's disease and to investigate other motor disorders. What will be the effect of these drugs on the mind and mood? Only a psychiatrist can hope to make use of this opportunity, for he, not a neurologist, is armed with the techniques and training necessary to evaluate the effects of drugs on the mind. In reverse, the motor effects of new antipsychotic drugs are of great interest and should be evaluated by a neurologist. Thus, each discipline is, for its own purpose, using tools which produce effects best exploited by the other. The almost exponential rate of growth of psycho- and neuropharmacology in the last decade suggests that the opportunities for collaboration between neurologist and psychiatrist in clinical pharmacology will accelerate in the future.

The outcome of collaboration between neurology and psychiatry is likely to be considerable, but, for historical reasons, it is often difficult to achieve. The organization of neurological services and psychiatric hospitals throughout the country has frequently led to their physical separation, with the result that each discipline acts in a visiting consultative role to its neighbour. This pattern is likely to change with the move of psychiatry into general and teaching hospitals, where opportunities for joint effort with neurology (and all other medical and surgical specialties) exist. Will there be psychiatrists interested in the mental sequelae of physical disability and brain diseases? Psychiatry itself may be separating more and more into its subspecialties-namely, child psychiatry, community psychiatry, addiction research units, psychogeriatrics, psychotherapy, behaviour therapy, etc. It is to be hoped that organic psychiatry, including neuropsychiatry, will evolve as one of the major recognized disciplines within psychiatry and will exploit the opportunities available for conjoint study of common problems.

C. D. MARSDEN

\section{REFERENCES}

Reid Report (1969). People with Epilepsy. Report of a Joint Sub-Committee of the Standing Medical Advisory Committee and Advisory Committee on the Health and Welfare of Handicapped Persons. DHSS. HMSO: London.
Symonds, C. (1970). Address to the Society of British Neurological Surgeons at its 81st Meeting 1970. Seacourt Press: Oxford. 